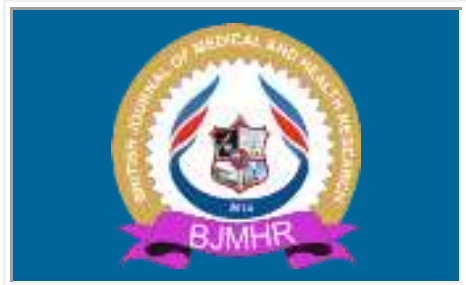

\title{
BJMHR
}

British Journal of Medical and Health Research

Journal home page: www.bjmhr.com

\section{Epidermolysis Bullosa Simplex: A Case Report}

\section{S.M. Biradar ${ }^{*}$, P. Keerthi sai ${ }^{1}$, H. Prudhvi raju', A. Harish kumar ${ }^{1}$, S C Marapur ${ }^{1}$, Vijaykumar Warad ${ }^{2}$, Shivakumar B' N V. Kalyane $^{1}$ \\ 1.Department of Clinical Pharmacy Practice, SSM College of Pharmacy and Research Centre, Vijaypur-586103. \\ 2.Department of Medicine, Shri B M. Patil Medical College Hospital and Research Centre, Vijaypur-586103.}

\section{ABSTRACT}

Epidermolysis bullosa (EB) is a rare and genetically determined skin fragility disorders. It has many genetic and symptomatic variations, but all share the prominent symptom of extremely fragile skin that blisters and tears from minor friction to trauma. It is always painful, often pervasive and debilitating. Its affects 1 out of every 50,000 live births and those born with it are often called 'Butterfly Children' that is delicate and fragile as butterfly wings. As there is no standard protocol for the treatment of EB and clinic features remains multiple and varied, hence treatment for the disease remains a major challenge. Therefore the current therapy focuses on prevention of progression of disease, nutritional support, bandage dressing and psychological support to the parents/guardians. Hence the platform provides this is a scope for discovery and development new drugs/treatment strategic plans for the treatment of Eidermolysis bullosa in order to recede the innocent babies' agony.

Keywords: Epidermolysis bullosa; Epidermolysis bullosa simplex; Multidisciplinary treatment; Scope for Gene therapy. 


\section{INTRODUCTION}

Epidermolysis bullosa (EB) is a heterogeneous group of rare genodermatoses characterized by cutaneous and/or mucosal fragility resulting in post-traumatic blistering. ${ }^{1}$ EB is traditionally categorized into four primary types based on the level of skin cleavage: simplex (EBS), junctional (JEB), dystrophic (DEB), and Kindler's syndrome. The severity ranges greatly from infant mortality to the presence of painful blisters and wounds on the hands and feet. ${ }^{2} \mathrm{~EB}$ is a rare disease; the incidence is approximately 1 in 50,000 live births, and the prevalence is 1:20,000-1:100,000 in the USA and Europe. ${ }^{3}$ Epidermolysis bullosa simplex (EBS) is a mechanobullous genodermatosis characterized by an intraepidermal split through the cytoplasm of the basal keratinocytes. Three main subtypes exist based on onset, severity and localization of blistering: EBS-localized (EBS-loc) with blistering confined to hands and feet from infancy, EBS-generalized, non-Dowling Meara (EBSgen) with early onset of generalized blistering, and EBS Dowling Meara (EBS-DM) with severe congenital circinate ('herpetiform') blistering often involving mucosal surfaces, development of palmoplantar keratoderma and the characteristic clumping of keratin filaments in skin samples visualised by electron microscopic (EM) analysis. In the majority of cases EBS is caused by autosomal dominant mutations in the KRT5 and KRT14 genes encoding the basal epidermal keratins 5 (K5) and 14 (K14), respectively. ${ }^{4}$ Immunofluorescence (IF), antigenic mapping, and transmission electron microscopy are key diagnostic tools used to confirm and identify a particular subtype of EB.

The current type of EBS was diagnosed by skin biopsy for immunofluorescent mapping, genetic testing and prenatal testing. Treatment options for patients with EB are limited. The primary aim of the treatment is to protect the skin, reduce the blistering, prevent the infection and its complications, and promote the wound healing. The prognosis of EB can vary dependent on the subtypes. While some patients have normal life expectancies, others can be at major risk of death during the first few years of life. For those who survive into adulthood, life can be severely limited. ${ }^{5}$

\section{Case Study}

A 6 days old neonate was hospitalized with complaints of fluid filled skin lesions over oral cavity, trunk and extremities since birth. The baby was born to non-consanguineous parents. The late pre-term (36-week 5 days) male baby with birth of $3.5 \mathrm{~kg}$ was born to 35 years old first gravida mother by caesarean section with uneventful perinatal period. There was no history of similar disorder in the families of either of the non-consanguineously married parents. Due to all lesions over oral cavity he is inability to breast feed. On examination of a baby it was observed that the Systemic examination was normal. The multiple bullae and 
erosions with areas of crusting present over trunk, B/L upper and lower extremities (Fig. No.1). Few erosions with whitish plaques present over buccal mucosa. Hyper pigmentation present over finger and toenails. Conjuctiva, cornea and scalp were normal. Nails were dystrophic (Fig. No. 2). Immunofluorescence antigen mapping (IFM) was performed and it reported frozen sections of patient's skin were stained with monoclonal antibodies against Type VII collagen, Type IV collagen and laminin 332. There were no epidermis in the section studied, stained with above antibodies on the dermal side. These features are highly suggestive of epidermolysis bullosa simplex. The final diagnosis of the disease was based on subjective clinical characteristics and immunofluorescence antigen mapping report as an Epidermolysis Bullosa Simplex. Based on the symptomatic treatment, Paraffin gauge dressing was applied on the wounds as lubrication, Tab. Bact (mupirocin) antibiotic ointment was applied topically for infection, Syrup. Calpol (Paracetamol) given $2 \mathrm{ml}$ twice a day for fever and pain, but not associated with chills and rigors.

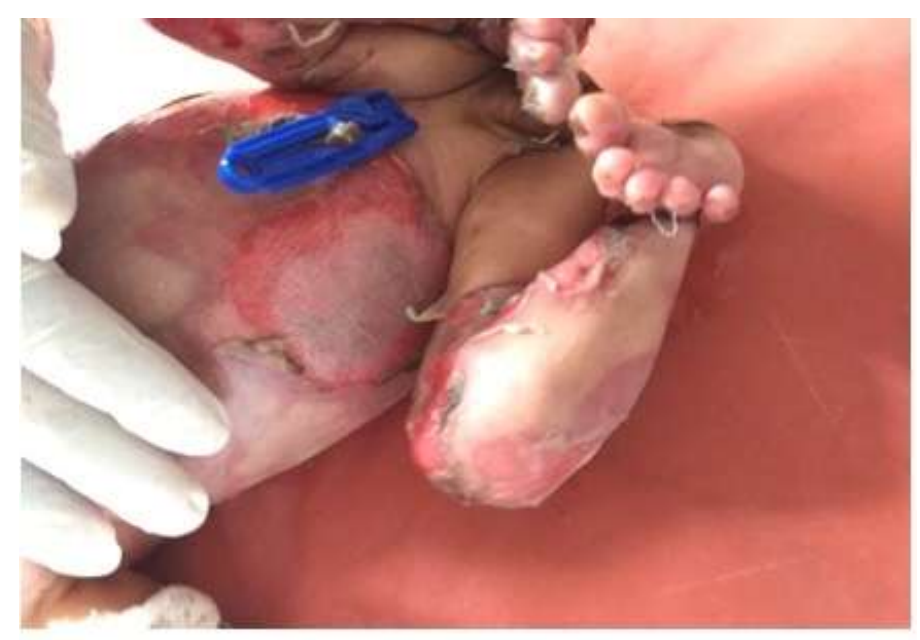

Figure 1: erosions and crusted lesions all over the body

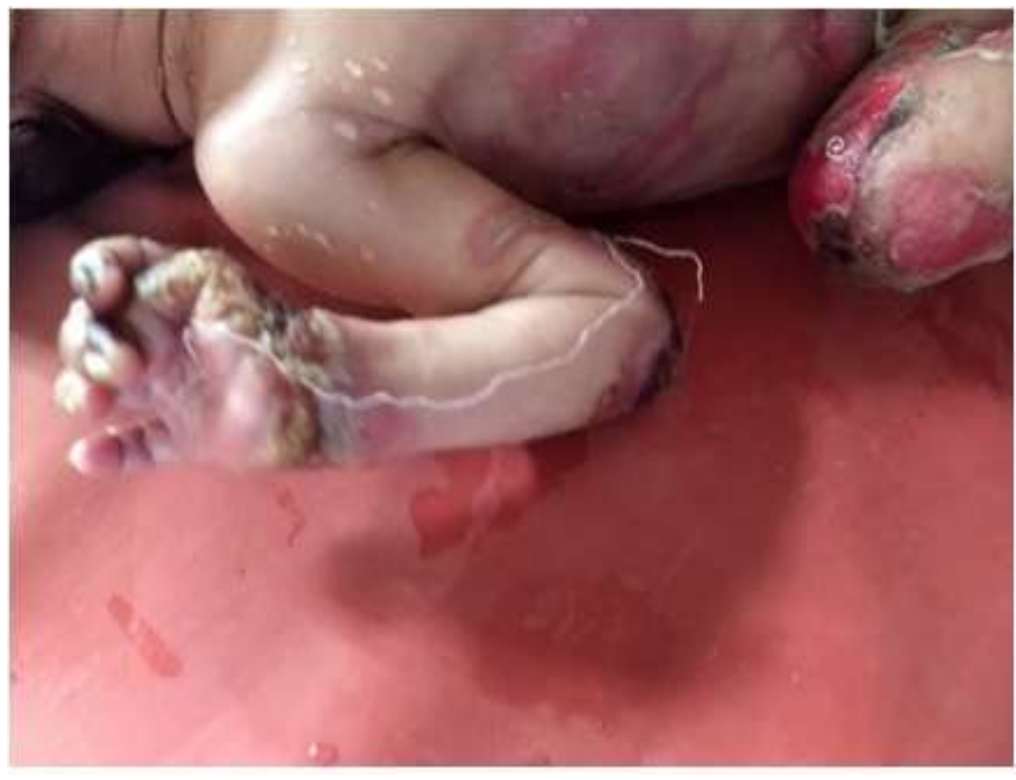

Figure 2: Dystrophic nails 


\section{DISCUSSION}

Epidermolysis bullosa (EB) comprises a group of genetically determined skin fragility disorders, which are characterized by blistering of the skin and mucosa, in response to little or no apparent trauma. These disorders represent heterogeneous phenotypes and are associated with a variable range of complications, from localized skin fragility to neonatal death. ${ }^{6}$ According to National Epidermolysis Bullosa Registry (NEBR), the prevalence and incidence of EB was estimated at approximately 8 per one million population and 19 per one million live births, in 1990 and 1986-1990, respectively. ${ }^{7}$ This disease was first to be explained by Von Hebra in 1870 as 'Erblichen Pemphigus', which was later modified to its recent name 'epidermolysis bullosa hereditaria' by Koebner in 1886. The first classification of the disease was done even much later in 1962 by Pearson. ${ }^{8}$

Epidermolysis bullosa simplex (EBS) is usually dominantly inherited, and involves disorders of the genes for Keratins 5 and 14 and plectin. Recently, several suprabasal types of EBS have been described as well. Blistering occurs within the uppermost layer of the skin, which is the epidermis. EBS may be localized to the hands and feet or there may be a generalized distribution, with relatively mild internal involvement. Those with EBS may have thickened calluses on the palms and soles, oral blistering during infancy and rough, thickened fingernails/toenails. EBS does not usually scar. There are rare recessive forms. EBS with plectin mutations may be associated with muscular dystrophy. ${ }^{9}$ However in the present case report symptoms of EBS are first seen at birth with multiple bullae and erosions with areas of crusting present over the trunk and bilateral upper and lower extremities. Few erosions with whitish plaques present over buccal mucosa. Hyperkeratosis present over finger and toenails. The blisters either occur spontaneously or in reaction to minor trauma. In the present case there was no family history of blistering disorders which points away from an autosomal dominant inheritance. There was no history of consanguinity amongst the parents which have been suggestive of an autosomal recessive inheritance. ${ }^{10}$ Hence, in this case It is due to mutation in the cytokeratin genes 5 (KRT5) or 14 (KRT14). These genes encode for intracellular proteins, K5 and K14 respectively, which are responsible for maintaining tissue integrity. They largely affect the mechanical integrity of the basal keratinocytes, which causes a substantial trauma to induce a blister.

There is presently no definitive cure for EB and the objective of treatment is to alleviate symptoms and provide supportive measures. Therapy is therefore focused on the prevention of lesions and its complications. Optimum management of this disease can only be achieved by a multidisciplinary team, which should include the following specialists: dermatologist, surgeon, nutritionist, dentist, physiotherapist, nurse, psychologist, pain specialist, and geneticist. The 
treatment plan must be individualized and optimal communication among team members is a vital factor in obtaining good results. A key to successful management is expert nursing care. Nursing the babies on thick foam pads protects them from undue trauma induced blistering. Special precautions need to be taken for older children in the use of adhesive tapes, sphygmomanometer cuffs, tourniquets and other instruments that cause shearing of skin or mucous membranes. The erosions should be cleaned with sterile normal saline and covered with non adherent dressings. Topical antibiotics are generally avoided because of the risk of emergence of antibiotic resistant bacteria. Oral and dental care should commence as soon as tooth eruption begins. Non-adhesive dressing pads or Vaseline impregnated gauze covered by soft, bulky dressings are ideal. Nutritional support is important for adequate growth and development and to promote optimal wound healing. To families of affected children, prenatal diagnosis using molecular techniques offers genetic counseling. ${ }^{11}$ The treatment plan must be individualized, and optimal communication among team members is a vital factor in obtaining good results. Similarly the current patient has been rightly treated as above in coordination between the multidisciplinary team in order to address the EBS.

\section{CONCLUSION}

As the EBS is inherited disease, there is no standard treatment protocol for the disease till today. Hence the treatment is challenging one and should be treated symptomatically on evidence based. Otherwise the disease may be the fatal one. Hence the therapy was focused on prevention of progression of skin lesions and inhibiting the further complications of the disease. Psychological support for parents and family members are important for emotional tolerability. Nutritional support is an important for adequate growth and development, to promote optimal wound healing. There is an urgent need for the development of genetically based new strategic plan for the treatment of EBS such as, gene therapy, stem cells transplantation and recombinant technology adaption in order to reduce the innocent babies' agony.

\section{REFERENCES}

1. Jennifer B, Christine C, Devos C et.al. Pain and quality of life evaluation in patients with localized epidermolysis bullosa simplex. Orphanet journal of rare diseases.2017;12(119):1-8.

2. Rebecca C B. Epidermolysis bullosa- An insider's perspective to a rare genetic connective tissue disorder. Journal of the dermatology nurses association.2016;8(1):4656.

3. Maldonado-colin G, Hernandez-zepeda C, Duran-mckinster C et.al. Inherited epidermolysis bullosa: A multisystem disease of skin and mucosae fragility. Indian journal of paediatric dermatology.2017;18(4):267-273. 
4. Bolling M C, Jongbloed J D H, Boven L G H, et.al. Plectin mutations in basal epidermolysis bullosa with wild-type KRT5 and KRT14 genes. University of Groningen.2010;82-85.

5. Caroline E F, Raphael AY, Thomas E S. Epidermolysis bullosa: a case report. Chronic wound care management and research.2018;5:17-21.

6. David J F, Epidemiology of inherited epidermolysis bullosa based on incidence and prevalence estimates from the national epidermolysis bullosa registry. JAMA Dermatol.2016;152(11):1231-1238.

7. Jo-David F. Inherited epidermolysis bullosa. Orphanet journal of rare diseases.2010;5(12):1-17.

8. https://rarediseases.org/rare-diseases/epidermolysis-bullosa/

9. Peterside O,Kunle-Olowu OE, Adeyemi OO et.al. Epidermolysis bullosa: a case report. Nigeria journal of paediatrics.2012;39(4):1-3.

10. Morelli JG. Vesiculobullous disorders. In Nelson Text Book of Pediatrics. 18th edition. Philadelphia, Pennsylvania, Saunders;2007:2685-93.

11. Dr. Purushotham D R, Dr. Sunil B, Dr. Adarsh E et.al. epidermolysis bullosa in newborn: a case report. Scholars journal of applied medical sciences.2014;2(1B):149151.

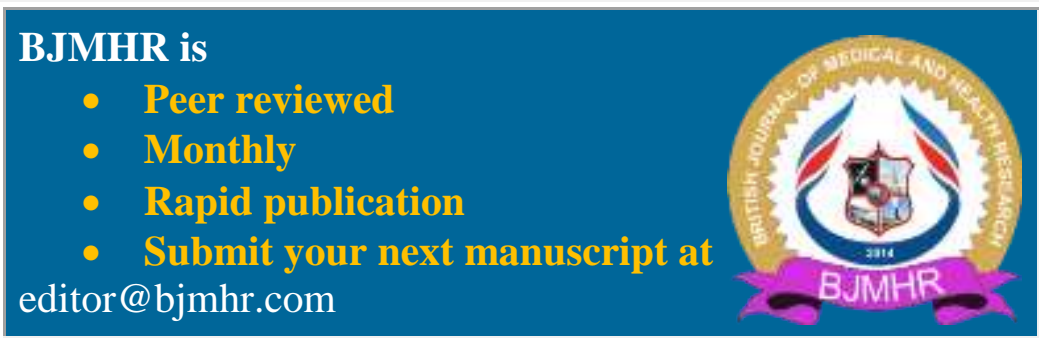

